\title{
Well-posedness, stability and conservation for a discontinuous interface problem: an initial investigation.
}

\author{
Cristina La Cognata and Jan Nordström
}

\begin{abstract}
A robust interface treatment for the discontinuous coefficient advection equation satisfying time-independent jump conditions is presented. The aim of the investigation is to show how the different concepts like well-posedness, conservation and stability are related. The equations are discretized using high order finite difference methods on Summation By Parts (SBP) form. The interface conditions are weakly imposed using the Simultaneous Approximation Term (SAT) procedure. Spectral analysis and numerical simulations corroborate the theoretical findings.
\end{abstract}

\section{Introduction}

In this paper we study fundamental properties such as well-posedness, stability and conservation for an advection equation, which changes wave-speed at the interface separating two spatial domains. The solution satisfies a time-independent jump-condition, which makes it discontinuous. The first goal is to show that for any piecewise constant advection velocity and interface jump condition the continuous problem is always well-posed. We provide a straightforward condition for checking conservation despite the presence of discontinuities. Applications where this is of interest include acoustic electromagnetism, seismology and fluid dynamics, [6],[10],[11],[12].

Stability and conservation at interfaces have also been studied in [2],[3],[4] for the case of identical velocities in the two domains. We extend this investigation by showing how well-posedness, conservation and stability are related in a more

Cristina La Cognata

Department of Mathematics, Computational Mathematics, Linköping University, SE-581 83

Linköping, Sweden, e-mail: cristina.la.cognata@liu.se

Jan Nordström

Department of Mathematics, Computational Mathematics, Linköping University, SE-581 83

Linköping, Sweden, e-mail: jan.nordstrom@liu.se 
general setting. SBP-SAT schemes, [13],[14], up to fifth order of accuracy are used to exemplify that the interface treatment is stable and accurate for all theoretically meaningful cases.

\section{The discontinuous interface problem}

Consider two advection equations with different real positive advection velocities, $a$ and $b$

$$
\begin{aligned}
& u_{t}+a u_{x}=0, x \leq 0, t \geq 0, \quad \text { with } \quad v(0, t)=c u(0, t), \\
& v_{t}+b v_{x}=0, x \geq 0, t \geq 0,
\end{aligned}
$$

where $c$ is a real constant which makes the solution discontinuous at the interface point $x=0$ when it is different from one.

\subsection{Well-posedness and conservation}

Proposition 1. The interface problem defined by the coupled equations (1) is wellposed for any positive $a, b$ and any constant $c \in \mathbb{R}$.

Proof. By applying the energy method to (1) using a modified $L^{2}$-norm we get

$$
\int_{-\infty}^{0} u\left[u_{t}+a u_{x}\right] d x+\int_{\infty}^{1} \alpha_{c} v\left[v_{t}+b v_{x}\right] d x=0
$$

where $\alpha_{c}$ is a positive free weight parameter. By ignoring the outer boundary terms, integration by parts leads to an energy estimate if $\alpha_{c}$ verifies

$$
-a+\alpha_{c} b c^{2} \leq 0
$$

Uniqueness of the solution can be proved by using the same technique. Existence can be proved by using the Laplace transform technique for the initial boundary value problem, see [5],[7] for details.

Proposition 2. The interface problem (1) is conservative if

$$
c=\frac{a}{b} .
$$

Proof. The weak formulation of (1) is given by

$$
\int_{-\infty}^{\infty}[\phi w]_{0}^{t} d x-\int_{-\infty}^{\infty} \int_{0}^{t}\left[\phi_{t}+\phi_{x} \bar{u}\right] w d x d t+\int_{0}^{t} \phi w[a-b c]_{x=0} d t=0 .
$$


where $\phi(x, t) \in C^{\infty}$ with compact support in the spatial interval $[-\infty, \infty]$ while $\bar{u}=a$ for $x \leq 0$ and $\bar{u}=b$ for $x>0$. Thus, all the terms at the interface vanish, resulting in a conservative problem if $c=a / b$.

\section{The semi-discrete approximation}

The first derivative in space is approximated using summation-by-parts (SBP) finite difference operators $u_{x} \approx D \mathbf{u}=P^{-1} Q \mathbf{u}$, introduced in [13],[14]. $\mathbf{u}$ is the discrete grid function approximating the solution. From now on we indicate the difference operator with $P_{l, r}^{-1} Q_{l, r}$, which are related to the left and right spatial intervals respectively. By ignoring the boundaries we can write the approximation of (1) together with the SAT procedure [3],[4], for interface conditions as

$$
\begin{aligned}
& \mathbf{u}_{t}+a P_{l}^{-1} Q_{l} \mathbf{u}=P_{l}^{-1} \sigma_{L}\left(c u_{N}-v_{0}\right) e_{N} \\
& \mathbf{v}_{t}+b P_{r}^{-1} Q_{r} \mathbf{v}=P_{r}^{-1} \sigma_{R}\left(v_{0}-c u_{N}\right) e_{0},
\end{aligned}
$$

where $e_{N}=(0, \ldots, 0,1)$ and $e_{0}=(1,0 \ldots, 0)$ have length of the left and right domain grid, respectively. (5) can be written in a compact matrix form as follows

$$
\left(\begin{array}{l}
\mathbf{u} \\
\mathbf{v}
\end{array}\right)_{t}=P^{-1} \tilde{Q}\left(\begin{array}{l}
\mathbf{u} \\
\mathbf{v}
\end{array}\right)
$$

where

$$
P=\left[\begin{array}{cc}
P_{l} & 0 \\
0 & P_{r}
\end{array}\right], \tilde{Q}=-Q_{\Lambda}+\Sigma, \text { and } Q_{\Lambda}=\left[\begin{array}{cc}
a Q_{l} & 0 \\
0 & b Q_{r}
\end{array}\right] .
$$

The penalty matrix $\Sigma$ which contains the penalties coefficients is zero everywhere except at the boundary and interface points

\subsection{Stability and conservation properties of the semi-discrete approximation}

Similar to the continuous case we apply the semi-discrete energy method to (5) to derive stability conditions. To do so we multiply (5) with $u^{T} P_{l}, v^{T} P_{r}$ from the left and we obtain

$$
\frac{d}{d t}\left[\|\mathbf{u}\|_{P_{l}}^{2}+\alpha_{d}\|\mathbf{v}\|_{P_{r}}^{2}\right]=\text { IT }
$$

where $\alpha_{d}$ is a positive weight (not necessarily the same as in the continuous case). IT is a quadratic form given by

$$
\mathrm{IT}=\left(\begin{array}{c}
u_{N} \\
v_{0}
\end{array}\right)^{T} H\left(\begin{array}{c}
u_{N} \\
v_{0}
\end{array}\right), \quad H=\left[\begin{array}{cc}
\left(-a+2 c \sigma_{L}\right) & -\left(\sigma_{L}+\alpha_{d} c \sigma_{R}\right) \\
-\left(\sigma_{L}+\alpha_{d} c \sigma_{R}\right) & \alpha_{d}\left(b+2 \sigma_{R}\right)
\end{array}\right] .
$$


We have an energy estimate if IT $\leq 0$, which require $\mathrm{H}$ to be a negative semi-definite matrix. Hence, we need a condition on $\sigma_{L}$ and $\sigma_{R}$ to ensure this. In the full paper [8] we prove

Proposition 3. The semi-discrete schemes (5) for the coupled advection equations (1) has a stable interface treatment when

$$
\begin{aligned}
\left(-a+2 c \sigma_{L}\right)+\alpha_{d}\left(b+2 \sigma_{R}\right) & \leq 0, \\
\left(-a+2 c \sigma_{L}\right) \alpha_{d}\left(b+2 \sigma_{R}\right)-\left(\sigma_{L}+\alpha_{d} c \sigma_{R}\right)^{2} & \geq 0 .
\end{aligned}
$$

One can also prove, see [8], that

Proposition 4. The conditions in (9) imply that $P^{-1} \tilde{Q}$ has eigenvalues with negative semi-definite real parts.

As in the continuous case we rewrite (5) in a weak formulation to derive the conservation condition. We obtain the following discrete conservation criteria

Proposition 5. The semi-discretization (5) with the continuous conservation condition (3) is a conservative approximation if

$$
\sigma_{R}=\sigma_{L}-b
$$

The conservative approximation requires a conservative continuous problem.

\section{The relation between stability and conservation}

In this section we present explicit stability condition for the penalty coefficients $\sigma_{L, R}$ for different type of continuous problems and approximations. All the conditions are algebraically derived from (9)

Proposition 6. Consider the most general well-posed interface problem without assuming any conservation conditions. The semi-discrete approximation (5) is stable for all parameters $a, b, c$ when the penalty coefficients $\sigma_{L}, \sigma_{R}$ satisfy

$$
\begin{gathered}
\sigma_{R} \leq \frac{-b}{2} . \\
\frac{b+\sigma_{R}-\sqrt{\left(b+2 \sigma_{R}\right)\left(b-\theta\left(\frac{a}{c}\right)\right)}}{\theta} \leq \sigma_{L} \leq \frac{b+\sigma_{R}+\sqrt{\left(b+2 \sigma_{R}\right)\left(b-\theta\left(\frac{a}{c}\right)\right)}}{\theta} .
\end{gathered}
$$

where $\theta=1 /\left(\alpha_{d} c\right) \geq b c /$ a must hold for real penalty coefficients. 
Proposition 7. The continuous conservation condition (3) leads to a stable semidiscrete approximation if the penalty parameters $\sigma_{L}, \sigma_{R}$ satisfy (11) and

$$
\frac{b+\sigma_{R}-\sqrt{b\left(b+2 \sigma_{R}\right)(1-\theta)}}{\theta} \leq \sigma_{L} \leq \frac{b+\sigma_{R}+\sqrt{b\left(b+2 \sigma_{R}\right)(1-\theta)}}{\theta} .
$$

with $\theta=b /\left(a \alpha_{d}\right) \geq 1$.

Note that conservation and stability are two independent properties of the approximation (5). We have a stable and non-conservative semi-discretization if the assumptions of Proposition 6 are satisfied. Note also that for one norm, the stability requirements in Proposition 6 also lead to conservation. That norm is given by $\alpha_{d}=b / a$.

Proposition 8. The conditions (3) and (10) lead to a stable and conservative scheme if

$$
\frac{b}{1-\sqrt{\theta}} \leq \sigma_{L} \leq \frac{b}{1+\sqrt{\theta}} \quad \text { with } \quad \theta=b /\left(a \alpha_{d}\right) \geq 1
$$

\section{Numerical results}

In order to show the effect of the interface treatment we must restrict ourselves to a finite spatial domain, we choose $[-1,1]$.

In Fig. 1 we show a few of the frames of the time-evolution of a conservative solution of (1), namely $\sin (4 \pi(-1+3 t))$. The wave propagates with velocity $a=2$ in the left domain, $b=1$ in right domain and jump condition $c=2$. The initial data is zero in both domains. The computations are done using RK4 in time and SBP84 in space, with $C F L=0.1$ and 300 grid points in each domain. The penalty $\sigma_{L, R}$ satisfy the conservative assumptions of Proposition 8.
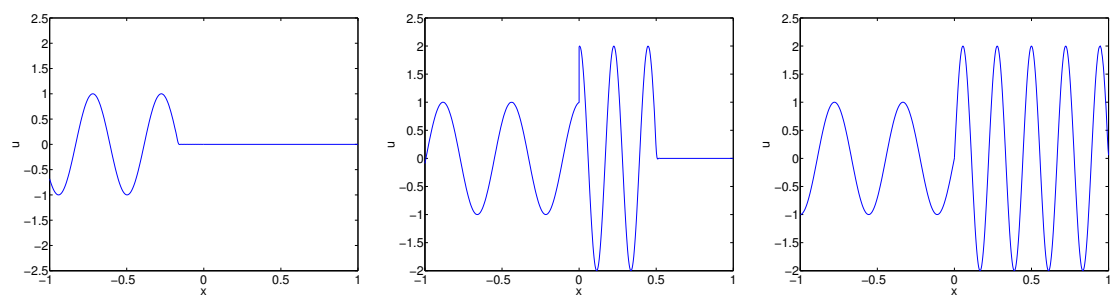

Fig. 1 Time-evolution of the wave function $\sin (4 \pi(-1+3 t))$ satisfying a conservative jump condition. The solution is computed with a conservation approximation (Proposition 7). Zero initial data in both domains and boundary condition given by $\sin (4 \pi(-1+3 t))$. The parameters are: $a=2$, $\mathrm{b}=1$ and $\mathrm{c}=2$. 


\subsection{Order of accuracy}

Next we establish the order of accuracy of our scheme by using the method of manufactured solutions with periodic boundary conditions. In Table 1 we present the accuracy of SBP21, SPB42, SBP63 and SBP84 operators in the $L^{2}$ norm for a nonconservative problem and approximation (stability conditions from Proposition 6). Table 1 shows that the solutions computed with the considered SBP operators converge with the correct $2^{\text {nd }}, 3^{\text {th }}, 4^{\text {th }}$ and $5^{\text {th }}$ order, respectively. We obtain analogous results for a conservative problem with both conservative and non-conservative approximation.

Table 1 Convergence rate as a function of grid $\mathrm{N}$ points for the non-conservative interface problem (1) and semi-discretization (5). $u_{l}$ and $u_{r}$ are the computed solutions in the left and the right domain rispectively. Parameters setting: $\mathrm{a}=3, \mathrm{~b}=2, \mathrm{c}=3$. Interface penalties $\sigma_{L, R}$ satisfying the stability conditions of Proposition 6.

\begin{tabular}{lllllllll}
\hline$L^{2}$ & SBP21 & & SBP42 & \multicolumn{2}{c}{ SBP63 } & \multicolumn{2}{c}{ SBP84 } \\
\hline $\mathrm{N}$ & $u_{l}$ & $u_{r}$ & $u_{l}$ & $u_{r}$ & $u_{l}$ & $u_{r}$ & $u_{l}$ & $u_{r}$ \\
\hline 80 & 2.0124 & 2.0267 & 3.0397 & 3.0096 & 3.6801 & 3.8770 & 4.5847 & 5.0897 \\
160 & 2.0086 & 2.0102 & 3.0713 & 3.0083 & 3.8480 & 4.0149 & 4.7510 & 5.0624 \\
320 & 2.0059 & 2.0044 & 3.0359 & 3.0068 & 3.9590 & 4.0052 & 4.9033 & 5.0176 \\
\hline
\end{tabular}

\subsection{The spectrum}

Given that our numerical scheme is accurate, we are now interested in showing that the interface treatment produces a negative semi-definite spectrum, which converges to the continuous spectrum. The semi-discrete spectrum is given by the eigenvalues of $P^{-1} \tilde{Q}$ defined in (6). The continuous spectrum of (1) is derived by using the Laplace Transform technique [5],[7] and is given by the infinite sequence

$$
s=\frac{a b}{a+b}[\log (|c d|)+2 i \pi k], \quad k \in \mathbf{Z} \quad \text { with } \quad c d \neq 0
$$

The real constant $d$ defines the boundary closure through $u(-1, t)-d v(1, t)=0$. In Fig. 2 we plot the semi-discrete spectrum vs the continuous one for a conservative problem and approximation. We can see that the spectra have eigenvalues with negative real parts, which implies well-posedness and a stable semi-discretization as stated in Proposition 4. We get similar plots for the other problems and schemes.

Table 2 show the order of convergence for the semi-discrete spectra to the continuous spectra for SBP21, SPB42, SBP63 and SBP84 operators. The convergence rate is computed by measuring the distance between the eigenvalues from the semidiscrete spectrum and the eigenvalues from the continuous spectrum. Note that Ta- 
Fig. 2 Continuous and semidiscrete spectrum of 4 th order SBP-SAT approximation. Penalty coefficients $\sigma_{L}, \sigma_{R}$ as in Proposition 7. Parameter setting: $a=2, b=1$, and $c=2$

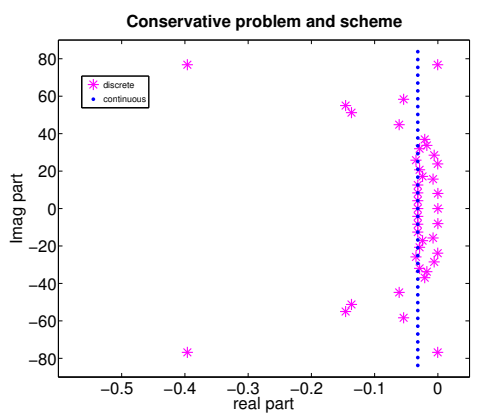

ble 2 show that the convergence is the same as the order of the internal approximation.

Table 2 Convergence rate as a function of $\mathrm{N}$ grid points for the non-conservative interface problem (1) and semi-discretization (5). Parameters setting: $a=3, b=2, c=3$. Interface penalties $\sigma_{L, R}$ satisfying the stability conditions of Proposition 5.

\begin{tabular}{lllll}
\hline $\mathrm{N}$ & SBP21 & SBP42 & SBP63 & SBP84 \\
\hline 40 & 2.4430 & 5.2086 & 6.1259 & 10.1153 \\
80 & 2.0485 & 4.2217 & 6.9556 & 8.9885 \\
160 & 2.0197 & 4.0813 & 5.9620 & 8.8797 \\
\hline
\end{tabular}

Fig. 2 also show that a few discrete eigenvalues are located to the right of the continuous spectrum. According to the definition of strict stability, [5],[7], the time growth rate of a strictly stable approximation is bounded by the growth rate of the corresponding continuous problem. Hence, we prefer that the eigenvalues of the semi-discrete spectrum lies on the left side of the continuous spectrum. By adding suitable artificial dissipation to the semi-discretization (5), we can move the discrete spectrum to the left side of the continuous one without reducing accuracy. Fig. 3 show a close-up of the spectrum of the conservative approximation of the same case with and without artificial dissipation. The semi-discrete eigenvalues on the right in Fig. 3 converge from the left side implying strict stability. For a discussion on how to build artificial dissipation operators for SBP operators without losing accuracy and stability, see [9].

\section{Conclusions}

We have presented a complete analysis of the discontinuous coefficient interface problem. We have shown that a such problem is always well-posed and we have investigated when it is conservative. We have derived a stable SBP-SAT scheme which can be made conservative or non-conservative depending on our choice. We 

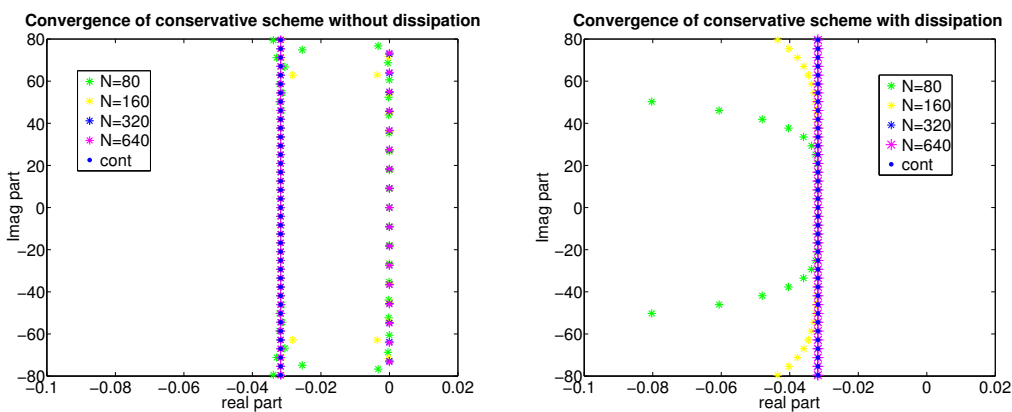

Fig. 3 Close-up of the spectra of the conservative problem plotted in Fig. 2 (left figure). The right fugure shows the same problem approximated by using artificial dissipation.

have also shown that the approximation can be made strictly stable by adding artificial dissipation without reducing the accuracy.

\section{References}

1. M. H. Carpenter, D. Gottlieb and S. Abarbanel. Time-stable boundary conditions for finitedifference schemes solving hyperbolic systems: Methodology and applications to high-order compact schemes. J. Compt. Phys., 129, pp:220-236 (1994).

2. M. H. Carpenter, J. Nordström, and D. Gottlieb. A stable and conservative interface treatment of arbitrary spatial accuracy. J. Comput. Phys., 148(2), pp:341-365 (1999).

3. M. H. Carpenter, D. Gottlieb, and S. Abarbanel. Revisiting and Extending Interface Penalties for Multi-domain Summation-by-Parts Operators J. Sci. Comput.,45, pp:118-150 (2010).

4. J.Gong, J. Nordström, Interface procedures for finite difference approximations of the advection-diffusion equation J. Comput. and Appl. Math. 236(5), pp:602-620 (2011).

5. B. Gustafsson, H.-O. Kreiss, A. Sundström. Stability theory of difference approximations for mixed initial boundary value problems II. Math. Computation, 26 (119) pp:649-686 (1972).

6. J. E. Kozdon, E. M. Dunham and J. Nordström. Simulation of Dynamic Earthquake Ruptures in Complex Geometries Using High-Order Finite Difference Methods, J. Sci. Comput., 50, pp:341-367 (2012) .

7. H.-O. Kreiss Stability theory of difference approximations for mixed initial boundary value problems I. Math. Computation 22 (104), pp:703-714 (1968).

8. C. La Cognata and J. Nordström. Well-posedness, stability and conservation for a discontinuous interface problem, ISRN: LiTH-MAT-R-2014/16-SE, Department of Mathematics, Linköping University.

9. K. Mattsson, M. Svärd and J. Nordström. Stable and Accurate Artificial Dissipation. J. Sci. Comput. Vol.21, No1, pp:57-79 (2004).

10. K. Mattsson and J. Nordström. High order finite difference methods for wave propagation in discontinuous media, J. Comput. Phys., 200, pp: 249-269. (2006).

11. J. Nordström, J. Gong, E. Van der Weide and M. Svärd. A stable and conservative high order multi-block method for the compressible Navier-Stokes equations, J. Comput. Phys.,228, pp:9020-9035 (2009).

12. J. Nordström and R. Gustafsson. High Order Finite Difference Approximations of Electromagnetic Wave Propagation Close to Material Discontinuities, J. Sci. Comput., Vol. 18, No. 2, pp:215-234 (2003). 
13. B. Strand. Summation by parts for finite difference approximation for $d / d x$. J. Comput. Phys., 110(1), pp:47-67, (1994).

14. M. Svärd, J. Nordström Review of summation-by-parts schemes for initial-boundary-value problems, J. Comput. Phys., 268, pp:17-38 (2014). 